\title{
Time course of sympathetic denervation of the rat ovary after freezing its nerve supply
}

\author{
W. R. Gibson, P. J. Roche, R. J. Summers* and S. N. Wylie \\ Department of Physiology, Monash University, Clayton, Victoria 3168, and ${ }^{*}$ Clinical Pharmacology \\ Unit, University of Melbourne, Department of Medicine, Austin Hospital, Heidelberg, Victoria 3084. \\ Australia
}

\begin{abstract}
Summary. Brief freezing of the ovarian vascular pedicle in rats reduced ovarian noradrenaline concentration, measured with HPLC, by $67 \%(P<0.01)$. Freezing of the ovarian suspensory ligament caused a $22 \%$ reduction $(P<0.01)$ and $33 \%$ reduction $(P<0.05)$ in 2 experiments. The numbers of adrenergic nerve terminals detected by fluorescence microscopy after these procedures were similarly reduced $(P<0.01)$. Freezing of both the pedicle and the ligament produced complete sympathetic denervation in about $50 \%$ of the ovaries. From Days 2 to 10 after operation no noradrenaline or nerve terminals were detected in 14 out of 27 ovaries. Nerve terminals were also eliminated from the oviduct. Reinnervation of the ovary began between Days 12 and 30 . It is concluded that the adrenergic innervation of the ovary is predominantly through nerves that accompany the vascular supply to the ovary and the ovarian suspensory ligament. Freezing of these routes is a simple and relatively atraumatic means of denervating the ovary for experimental studies.
\end{abstract}

\section{Introduction}

Although much is known of the distribution of sympathetic nerve terminals in the ovary, it has not yet been possible to ascribe any clearcut physiological function to them (for review, see Mohsin \& Pennefather, 1979). Sympathetic nerves would be expected to regulate blood flow through the ovarian blood vessels that they supply. In addition, they could have specific actions on follicles at any stage of their development or on corpora lutea. In attempts to test the possible roles of ovarian sympathetic nerves, numerous attempts have been made to denervate the ovary surgically, either by stripping the nerve bundles away from the blood vessels they accompany, or by severing the tissues containing the nerve bundles (Hill, 1962; Le Pere, Benoit, Hardy \& Goldzieher, 1966; Weiner, Wright \& Wallach, 1977; Lawrence \& Burden, 1980; Ojeda, Aguado \& Smith(White), 1983). A pharmacological approach, using 6-hydroxydopamine to deplete noradrenaline from nerve terminals, has also been used (Burden \& Lawrence, 1977).

In the present study we have applied a technique of briefly freezing the nerves to disrupt them, a modification of a technique used by Filogamo \& Muti (1968) to denervate loops of intestine. Sympathetic denervation was checked following the freezing of the ovarian vascular pedicle or the ovarian suspensory ligament, since these are reported to be the sole routes followed by sympathetic fibres innervating the ovary (Lawrence \& Burden, 1980). In additional experiments, both these structures were frozen, and the time course of the resulting denervation is described.

Part of this work has been reported in abstract form (Gibson, Roche, Summers \& Wylie, 1983).

\section{Materials and Methods}

General protocol. Denervation of the ovary was carried out in young adult (60-80 days), SpragueDawley rats on the day of metoestrus. Anaesthesia was produced by intraperitoneal injection of a 
mixture of methohexitone sodium (Brietal Sodium; Eli Lilly (Australia), Sydney), $50 \mathrm{mg} / \mathrm{kg}$, and amobarbital sodium (Amytal Sodium, Eli Lilly, Indianapolis, U.S.A.), $83 \mathrm{mg} / \mathrm{kg}$. Nerve fibres supplying the ovary were interrupted by briefly freezing them (Filogamo \& Muti, 1968).

One experimental series was designed to examine the relative contributions made by nerve fibres accompanying the ovarian artery and vein and by nerve fibres accompanying the ovarian suspensory ligament (Lawrence \& Burden, 1980) to the total sympathetic innervation of the ovary. In each rat, one ovary was exteriorized through a dorsal flank incision. The ovarian vascular pedicle, or the ovarian suspensory ligament, was frozen by touching them for $20( \pm 5) \mathrm{sec}$ with a stainless-steel probe taken from a bath of liquid nitrogen. The frozen regions were $1 \mathrm{~cm}$ from the ovary. After 7 days the rats were killed by overdose with i.p. pentobarbitone sodium (Euthatal; May \& Baker, West Footscray, Australia); the operated and contralateral (control) ovaries were removed and examined for sympathetic nerve terminals and assayed for noradrenaline as described below.

Another experimental series was designed to examine the time course of denervation. Procedures differed from the first series only in that the ovarian suspensory ligament and the ovarian vascular pedicle on the left side were frozen. The rats were killed at intervals from 1 to 30 days later. The oviducts as well as the ovaries were checked for the presence of nerve terminals.

Adrenergic innervation. Each ovary was cut into $15 \mu \mathrm{m}$-thick frozen sections and 5 representative sections were scored ('blind') for numbers of fluorescent nerve terminals revealed by the "SPG method" of de la Torre \& Surgeon (1976). These scores were combined to give a semi-quantitative 'nerve terminal index' for each ovary. This index could range from 0 , if no nerve terminal was found on any section, to 20 , if there were abundant nerve terminals on all 5 sections.

The remaining sections were combined and kept frozen until assayed for noradrenaline by high performance liquid chromatography and electrochemical detection (Howes, Summers, Rowe \& Louis, 1983): linear range, $20 \mathrm{pg}$ to $200 \mathrm{ng}$; sensitivity, $1 \mathrm{ng} / \mathrm{g}$ tissue.

The oviducts in the time course study were examined by the histofluorescence technique in the same way as the ovaries, except that 3 representative sections rather than 5 were examined.

Statistical analysis. Differences between means were tested using the Mann-Whitney U-test for unpaired data and the Wilcoxon matched-pairs signed-ranks test for paired data. Differences between frequencies in $2 \times 2$ tables were tested using Fisher's exact probability test (Siegel, 1956).

\section{Results}

Noradrenaline concentration and nerve terminals in unoperated ovaries

Each section taken from unoperated ovaries usually had about 200 '(moderate') to 500 ('abundant') fluorescent nerve terminals. These were associated with blood vessels and with antral follicles as well as other parts of the sections that were not readily identifiable under the fluorescence microscope. The semi-quantitative 'nerve terminal index', based on 5 sampled sections per ovary, had a mean \pm s.d. of $17.6 \pm 2.5(n=35)$ for unoperated ovaries in the first experimental series. The noradrenaline concentrations were similar in left and right ovaries and the overall mean \pm s.d. for the unoperated ovaries from all 5 experimental groups in this series was 248 $\pm 79(n=32) \mathrm{ng} / \mathrm{g}$.

\section{Freezing ovarian vascular pedicle or ovarian suspensory ligament}

Unilateral freezing of the ovarian vascular pedicle reduced the noradrenaline concentration of the ovary 7 days later by $67 \pm 9 \%$ (s.e.m.; 11 rats; $P<0.01$ ). The number of nerve terminals revealed by fluorescence microscopy was reduced $(P<0.01)$.

In 2 experiments, unilateral freezing of the ovarian suspensory ligament reduced the ovarian 
noradrenaline concentration by $22 \pm 7 \%$ (s.e.m.; 11 rats; $P<0.01)$ and by $33 \pm 11 \%$ ( 7 rats; $P<$ 0.05 ). The mean nerve terminal index was lower than in unoperated rats (N.S. and $P<0.01$ respectively in the 2 experiments). The results for nerve terminal index (semi-quantitative) generally paralleled those for noradrenaline concentration; the correlation coefficient based on all 54 ovaries of the main series was $0.68(P<0.001)$.

When there was substantial residual innervation (i.e. in all 'ligament-frozen' rats and a few 'pedicle-frozen' rats), the nerve terminals were distributed in the same way as in unoperated ovaries. When there was very little residual innervation (i.e. in some 'pedicle-frozen' rats) it was usually confined to the blood vessels.

The possibility that an operation on one side could affect the innervation of the ovary on the other side was checked by comparing the unoperated ovaries of these rats with the ovaries of wholly unoperated rats. There was no difference $(P>0 \cdot 1)$ in noradrenaline concentration or nerve terminal index for any of the operations.

Time course of denervation after freezing the ovarian suspensory ligament and ovarian vascular pedicle

Oestrous cycles recorded just before killing the rats were normal (usually 4 or 5 days). Ovarian weights were not affected by the denervation at any time; Day $1,43 \pm 3 \mathrm{mg}$ (operated side, mean \pm s.e.m., $n=5$ ) and $41 \pm 3 \mathrm{mg}$ (unoperated side); Days $2-10,39 \pm 2 \mathrm{mg}$ and $38 \pm 1 \mathrm{mg}(n=27)$; Days $12-30,40 \pm 2 \mathrm{mg}$ and $40 \pm 2 \mathrm{mg}(n=16)$.

On the first day after operation, the mean ovarian concentration of noradrenaline on the operated side had fallen to $9 \%(P<0.05)$ of that in the contralateral unoperated ovaries (Text-fig. 1a). Concentrations were always low on Days 2, 4, 7-8 and 10; in 14 of the 27 animals they were below the detection limit. Subsequently (Days 12, 16, 20 and 30), the concentrations were variable and higher than on Days $2-10(P<0.01)$ but still usually below unoperated levels (Text-fig. 1a).

Corresponding results for fluorescence microscopy of the same ovaries are shown in Text-fig. 1(b). The numbers of nerve terminals had fallen sharply on Day $1(P<0.01)$. From Days 2 to 10 , no nerve terminals were detected in most ovaries (22/26). From Days 12 to 30 terminals were more often present $(12 / 16 ; P<0.001$, Fisher's Exact Test for $2 \times 2$ tables) in variable numbers (Text-fig. 1b). In most ovaries any remaining terminals were found associated with blood vessels, antral follicles and other structures but they were sometimes confined to blood vessels if there were only very few of them.

The results for noradrenaline concentration and nerve terminal index were strongly related; $r=$ $0.81(n=77 ; P<0.001)$. Twenty-five ovaries were identified as denervated by absence of noradrenaline or absence of fluorescent nerve terminals, and for 16 ovaries $(64 \%)$ both methods agreed.

\section{Loss of nerve terminals in the oviduct}

Oviducts on the unoperated side contained nerve terminals associated with blood vessels and with the smooth muscle layers of the oviduct wall, especially that of the isthmus (in 3 out of 47 cases no terminal was seen, and in 3 others terminals were seen only in the muscle layers).

In 12 oviducts for which the ovarian suspensory ligament only was frozen, nerve terminals were invariably present, but the innervation (nerve terminal index) was reduced $(P<0.05)$ by $28 \%$. Freezing the ovarian vascular pedicle alone reduced $(P<0.01)$ the innervation by $64 \%$ and, in 6 out of 11 rats, produced complete denervation.

In the time-course series, freezing of the ovarian suspensory ligament and the ovarian vascular pedicle produced complete denervation at certain times. At Day 1 , all oviducts $(5 / 5)$ still had nerve terminals. From Days 2 to 10 there were usually (22/26) no terminals left, and from Days 12 to 30 terminals had reappeared in half $(8 / 16)$ the oviducts $(P<0.05$, Fisher's test $)$. The presence or absence of nerve terminals matched their presence or absence in the adjacent ovary $(24 / 26$ cases on Days 2-10). At Days 12-30, however, there were 5 rats (out of 16) in which the terminals had reappeared in the ovary while still absent from the oviduct. 


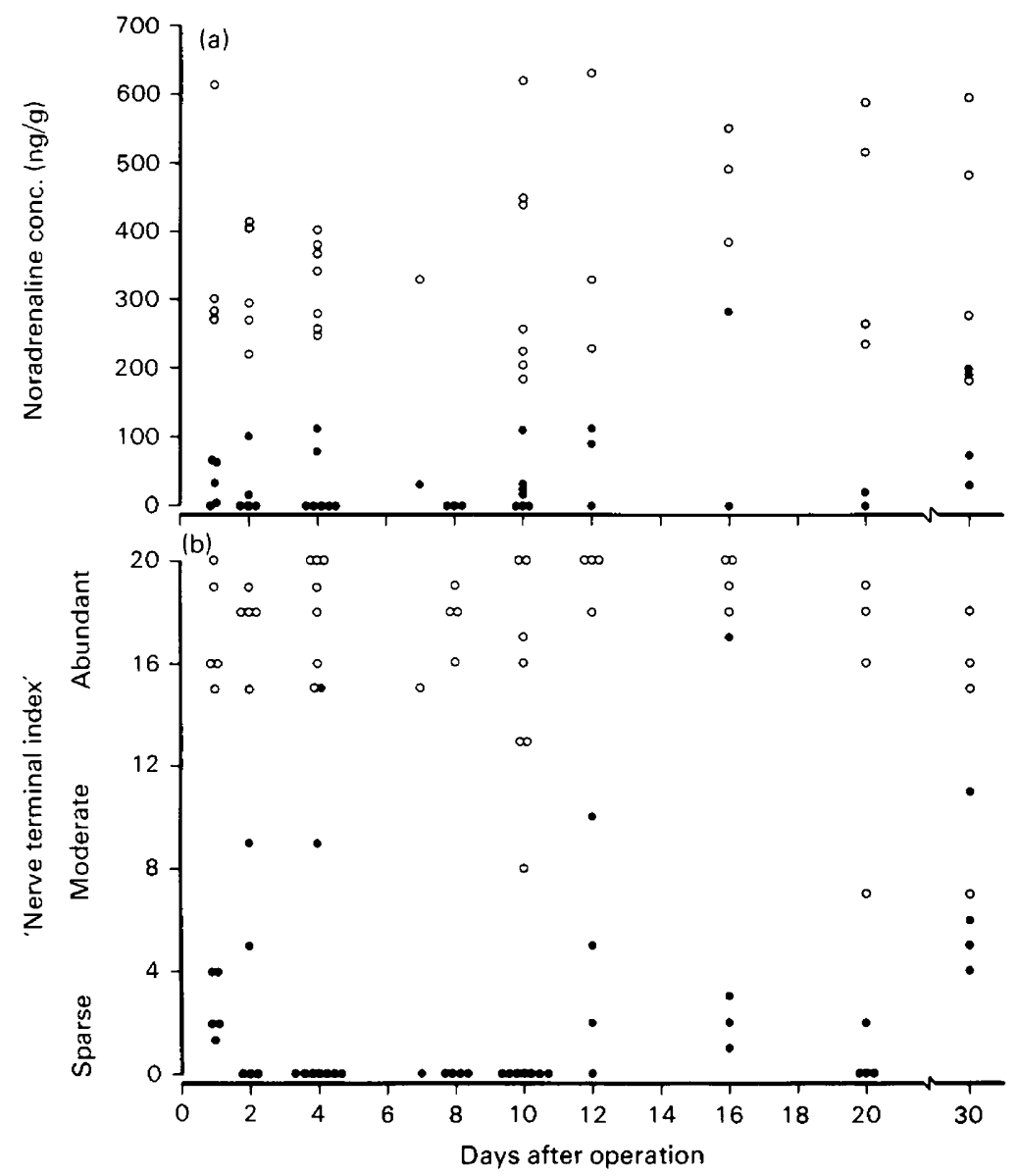

Text-fig. 1. Time course of denervation shown by (a) noradrenaline concentrations and (b) values for 'nerve terminal index' (see text), found by fluorescence microscopy, for ovaries at various times after unilateral freezing of the ovarian vascular pedicle and the ovarian suspensory ligament. Values for individual ovaries are shown. $\bigcirc$, Control ovaries;, , ovaries from the operated side.

\section{Discussion}

Our results show that, in the majority of rats, freezing the ovarian nerve supply eliminates noradrenergic nerves from the ovary for more than a week. Freezing has been used experimentally to destroy the sympathetic innervation of other tissues, including intestine (Filogamo \& Muti, 1968; Juorio \& Gabella, 1974), arteriole (Hirst, 1977) and costo-uterine muscle (Parkington \& Taylor, 1983), but this is the first application of the technique to the ovary. The freezing technique will offer a convenient and relatively atraumatic (Filogamo \& Muti, 1968) means of studying physiological functions in ovaries deprived of their sympathetic innervation. We observed no change in the appearance or the weights of the ovaries, which indicates that there was little or no oedema of the sort observed after cutting the ovarian plexus (Lawrence \& Burden, 1980).

However, our procedure may also have interrupted any ovarian sensory fibres and preganglionic parasympathetic fibres since these could be expected also to accompany the blood vessels supplying the ovary (Labate \& Reynolds, 1937; Burden \& Lawrence, 1978). Lawrence \& 
Burden (1980) severed the ovarian vascular pedicle and ovarian suspensory ligament. By freezing these two pathways we have confirmed their main finding that, in the majority of rats at least, the entire sympathetic innervation of the ovary arrives by these pathways. However, our observations are at variance with their conclusion that the majority of ovarian adrenergic nerves are derived from the nerve in the ovarian suspensory ligament, since our fluorescence microscopy and noradrenaline measurements both showed that nerves in the vascular pedicle contributed about twice as much to ovarian adrenergic innervation as did nerves in the ovarian suspensory ligament.

The variability amongst individual ovaries was striking because, in some cases, freezing the vascular pedicle alone produced complete sympathetic denervation and, in others, freezing of the pedicle and the ovarian suspensory ligament failed to do so. The contributions by the two pathways are probably variable, and some individuals may receive fibres from other pathways. Anatomical studies (Baljet \& Drukker, 1979) also indicate that this is likely. In physiological studies using the freezing technique it will be necessary to check for completeness of denervation.

Changes in the numbers of nerve terminals in the oviduct after freezing of the ovarian vascular pedicle, the ovarian suspensory ligament or both closely matched the changes in the adjacent ovary. It appears, then, that the oviduct also usually receives its entire sympathetic innervation by these two pathways, with the larger contribution being from the ovarian vascular pedicle.

We thank Mr Peter Rowe for carrying out most of the measurements of noradrenaline; many colleagues, especially Dr Brian Cragg, Miss Margaret Hartley, Dr Elspeth McLachlan, Dr Tim Neild, Dr Jo Pennefather, Dr Helena Parkington and Dr Graeme Taylor for helpful advice and discussion about the autonomic nervous system. The work was supported by Monash University Special Research Fund Grant Number M34/83.

\section{References}

Baljet, B. \& Drukker, J. (1979) The extrinsic innervation of the abdominal organs in the female rat. Acta anat. 104, 243-267.

Burden, H.W. \& Lawrence, I.E. (1977) The effect of denervation on compensatory ovarian hypertrophy. Neuroendocrinology 23, 368-378.

Burden, H.W. \& Lawrence, I.E. (1978) Experimental studies on the acetylcholinesterase-positive nerves in the ovary of the rat. Anat. Rec. 190, 233-242.

de la Torre, J.C. \& Surgeon, J.W. (1976) A methodological approach to rapid and sensitive monoamine histofluorescence using a modified glyoxylic acid technique: the SPG method. Histochemistry 49, 8193.

Filogamo, G. \& Muti, R. (1968) A propos d'une technique de dénervation du canal alimentaire au moyen du froid. C. r. Ass. Anat. $141873-879$.

Gibson, W.R., Roche, P.J., Summers, R.J. \& Wylie, S.N. (1983) Time course of denervation of the ovary after freezing its nerves. Proc. Aust. Soc. Reprod. Biol. 15, 23, Abstr.

Hill, R.T. (1962) Paradoxical effects of ovarian secretions. In The Ovary, 1st edn, Vol. II. ch. 16, pp. 231261. Ed. S. Zuckerman. Academic Press, New York.

Hirst, G.D.S. (1977) Neuromuscular transmission in arterioles of guinea-pig submucosa. J. Physiol, Lond. 273, 263-275.

Howes, L.G., Summers, R.J., Rowe, P.R. \& Louis, W.J. (1983) The simultaneous determination of 3,4-dihydroxyphenylethylene glycol, 3,4-dihydroxyphenylacetic acid and catecholamines in brain tissue by high performance liquid chromatography with electrochemical detection. Neurosci. Letters 38, 327-332.
Juorio, A.V. \& Gabella, G. (1974) Noradrenaline in the guinea pig alimentary canal: regional distribution and sensitivity to denervation and reserpine. $J$. Neurochem. 22, 851-858.

Labate, J.S. \& Reynolds, S.M. (1937) Sensory pathways of the ovarian plexus. Am. J. Obstet. Gynec. 34, 1-11.

Lawrence, I.E. \& Burden, H.W. (1980) The origin of the extrinsic adrenergic innervation to the rat ovary. Anat. Rec. 196, 51-59.

Le Pere, R.H., Benoit, P.E., Hardy, R.C. \& Goldzieher, J.W. (1966) The origin and function of the ovarian nerve supply in the baboon. Fert. Steril. 17, 68-75.

Mohsin, S. \& Pennefather, J.N. (1979) The sympathetic innervation of the mammalian ovary. A review of pharmacological and histological studies. Clin. exp. Pharmacol. Physiol. 6, 335-354.

Ojeda, S.R., Aguado, L.I. \& Smith(White), S. (1983) Neuroendocrine mechanisms controlling the onset of female puberty: the rat as a model. Neuroendocrino$\log y$ 37, 306-313.

Parkington, H. \& Taylor, G.S. (1983) The lack of postsynaptic responses following stimulation of the sympathetic fibres supplying the costo-uterine muscle of the guinea-pig. Pflügers Arch. 399, 290296.

Siegel, S. (1956) Nonparametric Statistics for the Behavioral Sciences. McGraw-Hill, Kogakusha, Tokyo.

Weiner, S., Wright, K.H. \& Wallach, E.E. (1977) The influence of ovarian denervation and nerve stimulation on ovarian contractions. Am. J. Obstet. Gynec. 128, $154-160$. 